\title{
Using P systems to Solve the Discrete Logarithm Problem used in Diffie-Hellman Key Exchange Protocol
}

\author{
Xiaojing Ma \\ Computer Department of Huazhong University of Science and Technology, Wuhan, Peoples Republic of China \\ lindahust@mial.hust.edu.cn \\ Zhitang Li and Hao Tu \\ Computer Department of Huazhong University of Science and Technology, Wuhan, Peoples Republic of ChinaEmail: \\ \{leeying, tuhao\}@mail.hust.edu.cn
}

\begin{abstract}
The discrete logarithm problem has been used as the basis of several cryptosystems, especially the DiffieHellman key exchange protocol. $P$ systems are a cluster of distributed parallel computing devices in a biochemical type. This paper presents a $P$ system with active membranes and strong priority to solve the discrete logarithm problem used in Diffie-Hellman key exchange protocol. To the best of our knowledge, it's the first time to solve the problem using $P$ systems.
\end{abstract}

Index Terms $-P$ systems, Discrete Logarithm Problem, Diffie-Hellman key exchange protocol

\section{INTRODUCTION}

Membrane computing (also called P systems) [1] was initiated in 1998 as a new class of distributed and parallel computing devices and much effort has been made on this research field [2]. In 2003, Thompson Institute for Scientific Information, ISI, has qualified the initial paper as "fast breaking" and the domain as "emergent research frontier in computer science". Membrane computing is inspired from the processes which take place in complex structure of a living cell. It has an attractive feature: parallelism.

The discrete logarithm problem plays an important role in cryptography. It has been used as the basis of several cryptosystems, such as the Diffie-Hellman key exchange protocol [3][9]. The problem used in Diffie-Hellman key exchange protocol can be formulated as: given a fixed primitive element $a$ of a finite field $F P(p)$ with a prime number $p$, and an integer number $b$, find the least positive integer $x$ such that, $a^{x} \equiv b(\bmod p) \quad 1 \leq x, b \leq p-1$.

However, no efficient algorithm for finding general discrete logarithms is known so far except for that in [4] which solves the problem on a quantum computer.

In this paper, we describe a $\mathrm{P}$ system with active

Manuscript received January 1, 2008; revised June 1, 2008; accepted July $1,2008$. membranes and strong priority to solve the discrete logarithm problem (DLP). To the best of our knowledge, it's the first time to solve DLP by membrane computing. The scheme we proposed in this paper can find the least positive index value $x$ for the DLP in less than $\lfloor\log (p-1)\rfloor$ loops.

We organize the remainder of this paper as follows. Section 2 describes the backgrounds of this paper, including the discrete logarithm problem used in DiffieHellman key exchange protocol, and $\mathrm{P}$ systems with active membranes and strong priority. $\mathrm{P}$ systems solving the Discrete Logarithm Problem are presented in section 3. Section 4 briefly gives the conclusion.

\section{BACKGROUNDS}

\section{A. THE DISCRETE LOGARITHM PROBLEM USED IN DIFFIE-HELLMAN KEY EXCHANGE PROTOCOL}

The Diffie-Hellman key exchange protocol [3][9] is used to build a secure 2-party key distribution channel to handle the problem of secure communications.

The protocol makes use of the conjectured intractability of the Discrete Logarithm Problem. The problem can be defined as follows.

Problem. Name: Discrete Logarithm Problem.

Instance: a finite field $G F(p)$ with a prime number $p$, a positive integer number $a$ which is a fixed primitive element of $G F(p)$, and a positive integer number $b$. $1 \leq a, b \leq p-1$.

$$
a^{x} \equiv b(\bmod p)
$$

Output: the least positive integer $x .1 \leq x \leq p-1$.

$$
x \equiv \log _{a} b \bmod p .
$$


A fixed primitive element for a finite field $F P(p)$ is the one whose powers build all the non-zero remainders modulo the prime number $p$. For example,

$3^{1} \bmod 7=3$

$3^{2} \bmod 7=2$

$3^{3} \bmod 7=6$

$3^{4} \bmod 7=4$

$3^{5} \bmod 7=5$

$3^{6} \bmod 7=1$

The set of all the results is $\{1,2,3,4,5,6\}$ which is the same to the set of all the non-zero integers modulo 7 . Thus, 3 is a fixed primitive element of the finite field FP(7).

Calculation of (1) is easy while calculation of (2) is much more difficult.

We discuss the Diffie-Hellman key exchange protocol [10] briefly as follows:

Each user choose a integer number $X_{i}$ from the set $\{1$, $2, \ldots, p-1\}$, and calculate

$$
Y_{i}=a^{X_{i}} \bmod p
$$

Each user keeps $X_{i}$ secret and makes $Y_{i}$ public.

When the users $U_{i}$ and $U_{j}$ need to communicate securely, they can calculate the secret key to protect the communication according to (4).

$$
K_{i j}=a^{X_{i} X_{j}} \bmod p
$$

The user $\mathrm{U}_{\mathrm{i}}$ can get $K_{i j}$ by computing

$$
K_{i j}=Y_{j}^{X_{i}} \bmod p=\left(a^{X_{j}} \bmod p\right)^{X_{i}}=a^{X_{i} X_{j}} \bmod p
$$

$\mathrm{U}_{\mathrm{j}}$ can get the secret communication key in a similar way. It is difficult for the other users to calculate the secret communication key of $\mathrm{U}_{\mathrm{i}}$ and $\mathrm{U}_{\mathrm{j}}$, since the problem to calculate $\log _{a} Y_{i} \bmod p$ and $\log _{a} Y_{j} \bmod p$ are both Discrete Logarithm Problem.

\section{B. P SYSTEMS}

$\mathrm{P}$ systems are a cluster of distributed parallel computing devices in a biochemical type, inspired by the structure and functioning of living cells [1][5]. The basic model consists of a hierarchical structure composed by several membranes. The essential ingredient of $\mathrm{P}$ systems is membrane structure. A membrane structure consists of several membranes which are hierarchically embedded in a main membrane. Each membrane delimits one region containing some objects. And a P system has evolution rules for objects and input-output prescription. An elementary membrane is one which does not contain other membranes. The space out of the membrane is called environment.

Starting from an initial configuration, the objects evolve according to the evolution rules. The evolution rules are inspired by the reactions which happen in a living cell. All the rules are applied in maximally parallel manner. That is, all the rules which can be applied must be applied, and all the objects which can involve must be involved.

We give an example of a general $P$ system.

$$
\begin{aligned}
& \Pi=\left(V, \mu, \omega_{1}, \ldots, \omega_{8},\left(R_{1}, \rho_{1}\right),\left(R_{2}, \rho_{2}\right), \ldots,\left(R_{8}, \rho_{8}\right), 8\right) \\
& V=\{a, b, d, e, f, g, h, s\} \\
& \left.\left.\left.\mu={ }_{1}\left[_{2}{ }_{3}\left[_{7}\right]_{7}[]_{8}\right]_{8}\right]_{3}[]_{4}\right]_{2}\left[_{5}\right]_{5}\left[{ }_{6}\right]_{6}\right]_{1} \\
& \omega_{3}=\left\{a^{4}, b^{3}, f\right\} \\
& \omega_{5}=\{h, s\} \\
& \omega_{7}=\{g, d\} \\
& \omega_{1}=\omega_{2}=\omega_{4}=\omega_{6}=\omega_{8}=\lambda \\
& R_{3}=\left\{a \rightarrow a\left(e, i n_{8}\right), r_{1}: b f \rightarrow f, r_{2}: f \rightarrow \delta\right\} \\
& \rho_{3}=\left\{r_{1}>r_{2}\right\} \\
& R_{1}=R_{2}=R_{4}=R_{5}=R_{6}=R_{7}=R_{8}=\varnothing \\
& \rho_{1}=\rho_{2}=\rho_{4}=\rho_{5}=\rho_{6}=\rho_{7}=\rho_{8}=\varnothing
\end{aligned}
$$

(1) $V$ is the alphabet of all the objects used in the P system.

(2) $\mu$ expresses the membrane structure of the $\mathrm{P}$ system. The membrane structure of this $\mathrm{P}$ system can be illustrated in Fig. 1, where the membrane structure can be expressed in the form of $\left.{ }_{1}\left[{ }_{2}\left[{ }_{3}[7]_{7}[8]_{8}\right]_{3}[4]_{4}\right]_{2}[5]_{5}[6]_{6}\right]_{1}$ and Fig. 2 too.

(3) $\omega_{i}, 1 \leq \mathrm{i} \leq 8$ is strings from $\mathrm{V}^{*}$ representing the set of objects present in region $i$. In Fig. 1, $a^{4}$ indicates that there are four occurrences of object $a$ in membrane with label " 3 ".

(4) $\mathrm{R}_{\mathrm{i}}, \quad 1 \leq \mathrm{i} \leq 8$ is the set of evolution rules associated with the region $i$. In $\mathrm{R}_{3}$, there are a evolution rule $a \rightarrow a\left(e, i n_{8}\right)$ which produce an objects $a$ and sends an object $e$ to the region 8 from one object $a$. Each paired objects $b$ and $f$ evolve to one object $f$ under $b f \rightarrow f$. $f \rightarrow \delta$ makes the object $f$ disappeared and dissolves the membrane.

(5) $\rho_{i} 1 \leq \mathrm{i} \leq 8$ is the set of priority relationship over $\mathrm{R}_{\mathrm{i}} \cdot \rho_{3}=\left\{r_{1}>r_{2}\right\}$ means that the rule $\mathrm{r}_{1}$ : $b f \rightarrow f$ has priority over the rule $\mathrm{r}_{2}: f \rightarrow \delta$. Firstly, the object $f$ will evolve according to $r_{1}$. The rule $r_{2}$ will not be used unless $r_{1}$ cannot be used.

(6) The " 8 " in $\Pi$ means the output region of the $P$ system, and the result of the computation is collected in the output region. In this example, the output is the number of objects present in the region 8 .

This $\mathrm{P}$ system can calculate $4^{2}$. We explain the computation procedure as follows:

In the initial configuration, there are four occurrences of object $a$, three occurrences of object $b$ and an object $f$. 
At the first step, the rule $b f \rightarrow f$ "absorbs" one object $b$ with the help of the object $f$, and $a \rightarrow a\left(e, i n_{8}\right)$ produces four objects $e$ and sends them to the region 8 .

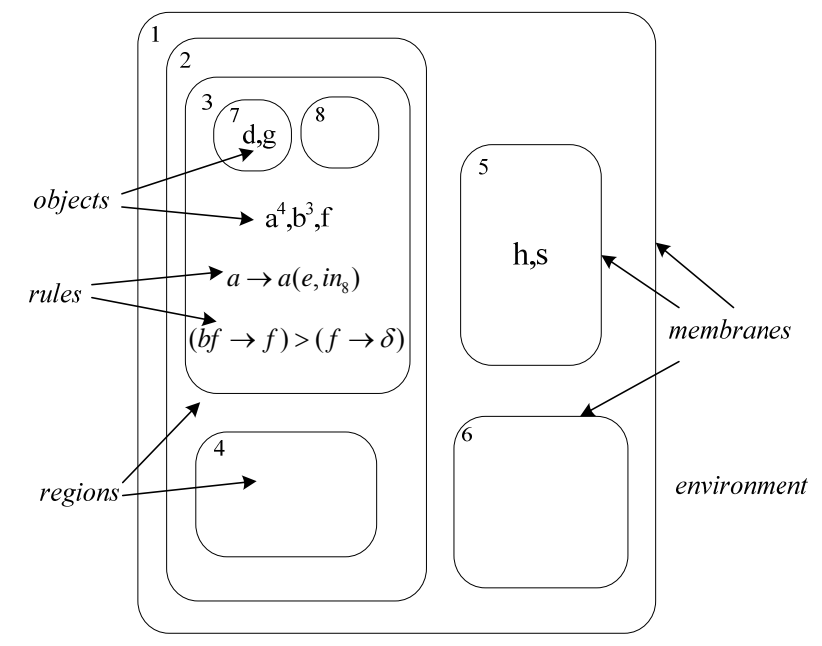

Figure 1. An example of membrane structure

The rule $f \rightarrow \delta$ is not used according to $(b f \rightarrow f)>(f \rightarrow \delta)$.

At the second step, $b f \rightarrow f$ "absorbs" one another object $b$ with the help of the object $f$, and $a \rightarrow a\left(e, i n_{8}\right)$ produces another four occurrences of object $e$. Now there are eight occurrences of object $e$ in region 8 .

The third step is similar to the second step, and there are twelve occurrences of object $e$ in the region 8 .

At the fourth step, $a \rightarrow a\left(e, i n_{8}\right)$ produces another four objects $e$ and sends them to the region 8 . There are sixteen $\left(4^{2}\right)$ occurrences of object $e$ in the region 8 . At the same time, there is no object $b$ left in the region 3 , so $b f \rightarrow f$ cannot be used. Thus, the rule $f \rightarrow \delta$ is used to dissolve the membrane with label " 3 ". There is no rule in the region 2 , so the computation is completed.

The hierarchical structure of membranes can also be presented by a rooted tree, as shown in Fig.2 which describes the membrane structure from Fig.1.

\section{P SYSTEMS WITH ACTIVE MEMBRANES}

There are many variants of $\mathrm{P}$ systems, one of which is $\mathrm{P}$ systems with active membranes [6][7]. P systems with active membranes are obtained by including rules for membrane division.

On the other hand, noting the fact that certain reactions are more active than others in a living cell, [1][8] consider a priority relationship on the set of rules in a given region.

Now, we describe the $\mathrm{P}$ systems with active membranes and priority. It is a construct expressed in (6).

$$
\Pi=\left(V, H, \mu, \omega_{1}, \ldots, \omega_{m}, R, \rho, e\right)
$$

(1) $V$ is a alphabet which contains all the alphabet used in the P system;

(2) $H$ is a set of labels for membranes.

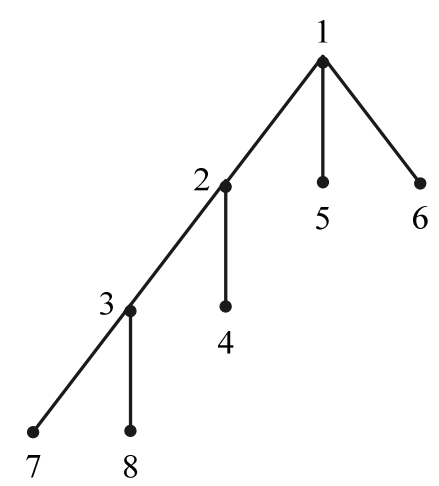

Figure 2. The tree describing the membrane structure from Fig.2.

(3) $\quad \mu$ is the initial membrane structure consisting of $m$ membranes.

$\omega_{1}, \ldots, \omega_{m}$ are strings over $V$, describing the objects placed in the $m$ regions of $\mu$.

(5) $R$ is a finite set of evolution rules, including rules for membrane division, of the following forms

( for $h_{0}, h_{1}, h_{2}, \in \mathrm{H}, \alpha_{1}, \alpha_{2}, \alpha_{3} \in\{+,-, 0\}$,

$\mathrm{x}, \mathrm{y} \in \mathrm{V}^{*}, \mathrm{a}, \mathrm{b}, \mathrm{c} \in \mathrm{V}$ ):

i. $\quad\left[{ }_{h} \mathrm{x} \rightarrow y\right]_{h}^{\alpha}$

(Object evolution rules, all the object pairs that can form string $x$ are replaced by the corresponding object pairs in string $y$.)

ii. $\mathrm{a}\left[{ }_{h}\right]_{h}^{\alpha_{1}} \rightarrow\left[{ }_{h} b\right]_{h}^{\alpha_{2}}$

(Communication rules, object $a$ is sent into the membrane, simultaneously, $a$ evolves to object $b$ and the charge of the membrane can be modified.)

iii. $\quad\left[{ }_{h} \mathrm{a}\right]_{h}^{\alpha_{1}} \rightarrow\left[{ }_{h}\right]_{h}^{\alpha_{2}} b$

(Communication rules, object $a$ is sent out of the membrane, modified to $b$ and the charge of the membrane can be modified too.)

iv. $\left[{ }_{h} \mathrm{a}\right]_{h}^{\alpha} \rightarrow b$

(Membrane dissolving rules, the membrane is dissolved, $a$ evolves to object $b$ )

v. $\quad\left[{ }_{h} \mathrm{a}\right]_{h}^{\alpha_{1}} \rightarrow\left[{ }_{h} b\right]_{h}^{\alpha_{2}}\left[{ }_{h} c\right]_{h}^{\alpha_{3}}$

(Elementary membrane division rules, the membrane is divided into two membranes with the same label, maybe of different charge; object $a$ is placed into two new membranes maybe replaced by new objects $b$ and $c$; each new membrane has a copy of all the other objects.)

vi. $\quad\left[h_{h_{0}}\left[h_{h_{1}}\right]_{h_{1}}^{+}\left[{ }_{h_{2}}\right]_{h_{2}}^{-}\right]_{h_{0}}^{0} \rightarrow\left[{ }_{h_{0}}\left[{ }_{h_{1}}\right]_{h_{1}}^{0}\right]_{h_{0}}^{0}\left[{ }_{h_{0}}\left[{ }_{h_{2}}\right]_{h_{2}}^{0}\right]_{h_{0}}^{0}$ (Membrane division rules for nonelementary membranes, membrane $h_{0}$ has 
two membranes of opposite charge which are separated into two new membranes with label " $\mathrm{h}_{0}$ "; and they have neutral charge.) $\rho$ is a set of priority relations for evolution rules. There are two types of priority relation: weak priority and strong priority. For rules with strong priority, if a rule $\mathrm{R}_{1}$ has priority over a rule $R_{2}$, and $R_{1}$ can be applied, then $R_{2}$ can not be used, regardless of whether there are objects suitable for $\mathrm{R}_{2}$.

For example, if $\mathrm{R}_{1}: \quad\left[{ }_{1} F F \rightarrow \lambda\right]_{1}^{0}$ and $\mathrm{R}_{2}:\left[{ }_{1} F G \rightarrow H\right]_{1}^{0}$, the current multiset is FFFG in membrane " 0 " of neutral charge, then rule $\mathrm{R}_{1}$ can be used, but $\mathrm{R}_{2}$ cannot be used even there are objects FG suitable for $\mathrm{R}_{2}$. On the contrary, $\mathrm{R}_{2}$ can be used if the relationship between $R_{1}$ and $R_{2}$ is a weak priority.

(7) $\mathrm{e} \in \mathrm{V}$. The number of the copies of object $e$ sent out to the environment is the computation result.

\section{SOlVING THE DisCRETE LOGARITHM PROBLEM USING P SYSTEM}

We discuss in this Section on how to apply P systems to solve the DLP. Recall the formulation of DLP in section 1. We wish to solve the problem shown in (1).

We construct a $\mathrm{P}$ system with active membranes and strong priority.

$$
\begin{aligned}
& \Pi=\left(V, H, \mu, \omega_{1}, \omega_{2}, \omega_{3}, R, \rho, X\right) \\
& V=\left\{A, B, C, D, F, G, H, H^{\prime}, X, \lambda, Z, Z^{\prime}, Z^{\prime \prime}\right\} \cup \\
& \quad\left\{k_{i}, k_{i}^{\prime}, k_{i}^{\prime \prime}, t_{i}, f_{i} \mid 1 \leq i \leq\lfloor\log (p-1)\rfloor\right\} \\
& H=\{1,2,3\} \\
& \left.\left.\mu=\left[{ }_{1}\left[{ }_{2}\right]_{3}\right]_{3}^{0}\right]_{2}^{0}\right]_{1}^{0} \\
& \omega_{1}=\left\{C, X, G^{b}\right\} \cup\left\{k_{i}^{\prime \prime} \mid 1 \leq i \leq\lfloor\log (p-1)\}\right. \\
& \omega_{2}=\left\{A^{a}\right\} \\
& \omega_{3}=\varnothing \\
& R=\{ \\
& R_{0}: A\left[{ }_{3}\right]_{3}^{0} \rightarrow\left[{ }_{3} A^{\prime}\right]_{3}^{0} \\
& R_{1}:\left[{ }_{3} k_{i}^{\prime \prime}\right]_{3}^{0} \rightarrow\left[{ }_{3} k_{i}^{\prime}\right]_{3}^{0} \\
& R_{2}:\left[{ }_{3} k_{i}^{\prime}\right]_{3}^{0} \rightarrow\left[{ }_{3} k_{i}\right]_{3}^{0} \text { for } 1 \leq i \leq\lfloor\log (p-1)\rfloor \\
& R_{3}:\left[{ }_{3} A^{\prime}\right]_{3}^{0} \rightarrow\left[{ }_{3} B D\right]_{3}^{0} \\
& R_{4}:\left[{ }_{3} B B C \rightarrow C\right]_{3}^{0} \\
& R_{5}:\left[{ }_{3} B C \rightarrow C\right]_{3}^{0} \\
& R_{6}:\left[{ }_{3} D\right]_{3}^{0} \rightarrow\left[{ }_{3} D E\right]_{3}^{0}
\end{aligned}
$$

$$
\begin{aligned}
& R_{7}:\left[{ }_{3} k_{i}\right]_{3}^{0} \rightarrow\left[{ }_{3} t_{i}\right]_{3}^{+}\left[{ }_{3} f_{i}\right]_{3}^{-} \text {for } 1 \leq i \leq\lfloor\log (p-1)\rfloor \\
& R_{8}:\left[{ }_{3} C t_{i} \rightarrow C Z^{\prime \prime}\right]_{3}^{+} \quad \text { for } 1 \leq i \leq\lfloor\log (p-1)\rfloor \\
& R_{9}:\left[{ }_{3} X \rightarrow X X\right]_{3}^{+} \\
& R_{10}:\left[{ }_{3} C f_{i} \rightarrow C X Z^{\prime \prime}\right]_{3}^{-} \quad \text { for } 1 \leq i \leq\lfloor\log (p-1)\rfloor \\
& R_{11}:\left[{ }_{3} X \rightarrow X X\right]_{3}^{-} \\
& R_{12}:\left[{ }_{3} D \rightarrow \lambda\right]_{3}^{+} \\
& R_{13}:\left[{ }_{3} D \rightarrow \lambda\right]_{3}^{-} \\
& R_{14}:\left[{ }_{3} E \rightarrow F\right]_{3}^{+} \\
& R_{15}:\left[{ }_{3} E \rightarrow F^{a}\right]_{3}^{-} \\
& R_{16}:\left[{ }_{3} k_{i} \rightarrow k_{i}^{\prime \prime}\right]_{3}^{+} \quad \text { for } 1 \leq i \leq\lfloor\log (p-1)\rfloor \\
& R_{17}:\left[{ }_{3} k_{i} \rightarrow k_{i}^{\prime \prime}\right]_{3}^{-} \quad \text { for } 1 \leq i \leq\lfloor\log (p-1)\rfloor \\
& \left.R_{18}:\left[{ }_{2}\left[{ }_{3}\right]_{3}^{+}\left[{ }_{3}\right]_{3}^{-}\right]_{2}^{0} \rightarrow{ }_{2}\left[{ }_{3}\right]_{3}^{0}\right]_{2}^{0}\left[{ }_{2}\left[{ }_{3}\right]_{3}^{0}\right]_{2}^{0} \\
& R_{19}:\left[{ }_{3} F^{p} \rightarrow \lambda\right]_{3}^{0} \\
& R_{20}:\left[{ }_{3} Z^{\prime \prime} \rightarrow Z^{\prime}\right]_{3}^{0} \\
& R_{21}:\left[{ }_{3} \mathrm{FG} \rightarrow \mathrm{H}\right]_{3}^{0} \\
& R_{22}:\left[{ }_{3} Z^{\prime} \rightarrow Z\right]_{3}^{0} \\
& R_{23}:\left[{ }_{3} G Z \rightarrow G\right]_{3}^{0} \\
& R_{24}:\left[{ }_{3} F Z \rightarrow F\right]_{3}^{0} \\
& R_{25}:\left[{ }_{3} H Z\right]_{3}^{0} \rightarrow \lambda \\
& R_{26}:\left[{ }_{3} H \rightarrow G F\right]_{3}^{0} \\
& R_{27}:\left[{ }_{3} F\right]_{3}^{0} \rightarrow\left[{ }_{3}\right]_{3}^{0} \mathrm{~A} \\
& R_{28}:\left[{ }_{2} X\right]_{2}^{0} \rightarrow\left[{ }_{2}\right]_{2}^{0} X \\
& R_{29}:\left[{ }_{1} X\right]_{1}^{0} \rightarrow\left[{ }_{1}\right]_{1}^{0} X \\
& \text { \} } \\
& \rho=\left\{R_{4,5}>R_{7}, R_{4}>R_{5}, R_{23,24}>R_{25},\right. \\
& R_{25}>R_{26}, R_{19}>R_{21}>R_{24}>R_{28} \text {, } \\
& \left.R_{19 \sim 27}>R_{1}, R_{2}\right\}
\end{aligned}
$$

For every prime number $p$ $\Pi=\left(V, H, \mu, \omega_{0}, \omega_{1}, \omega_{2}, R, \rho\right)$ is a deterministic $\mathrm{P}$ system and the evolution generated by this system will stop after several steps. In the last step, the number of object $X$ sent to the environment is the index $x$. We prove this by describing the process of evolution generated by $\Pi=\left(V, H, \mu, \omega_{0}, \omega_{1}, \omega_{2}, R, \rho\right)$.

The main idea of this $\mathrm{P}$ system to solve DLP is enumerating the value of index. Using membrane division, the system can check $2^{n}$ values of the index $\left(1 \sim 2^{\mathrm{n}}\right)$ in $n$ loops. Each loop can be divided into three stages. We generate enough objects $F$ at stage one; In stage two we get the remainder whose value is equal to the number of the object $F$ left after using $\mathrm{R}_{21}$. And in stage three the system checks if the remainder is equal to 
$b$. If it is equal to $b$, then the object $X$ will be output to the environment, otherwise, we go to the next loop.

Let us take a closer look at how one step of the loop is performed from the initial configuration. The process with only those critical rules and key objects is briefly demonstrated in Fig. 2.

In the initial configuration, the number of the object $G$ in membrane " 3 " corresponds to the modular $b$. The number of the object $X$ corresponds to the index $x$ whose value is one in the initial configuration. The number of the object $A$ in membrane " 2 " corresponds to the element a. Moreover, the objects $k_{i}(1 \leq i \leq\lfloor\log (p-1)\rfloor)$ in membrane " 3 " are used as counters, which control the membrane division.

According to the priority relationship $\mathrm{R}_{4,5}>\mathrm{R}_{7}$, the rules $\mathrm{R}_{0} \sim \mathrm{R}_{6}$ are used. The objects $A$ of number $a$ are sent into the membrane " 3 ", and we get the objects $E$ of the number $a^{2}$. Simultaneously, by using the rule $\mathrm{R}_{7}$, the "electrically neutral" membrane " 3 " is divided into two separate copies of opposite charge. Further, the object $k_{i}$ evolves to $t_{i}$ and $f_{i}$ with $t_{i}$ placed into the copy of positive charge and $f_{i}$ placed into the copy of negative charge respectively. The number of the object $k_{i}$ is $\lfloor\log (p-1)\rfloor$ corresponding to the number of loop times.

Next, $R_{8} \sim R_{18}$ are all applied in one step. $R_{12}$ and $R_{13}$ consume all the objects $D . \mathrm{R}_{8} \sim \mathrm{R}_{11}, \mathrm{R}_{14}$, and $\mathrm{R}_{15}$ generate $a^{2}$ objects $F$ and 2 objects $X$ in membrane of label " 1 ", with positive charge, correspondingly, $a^{3}$ objects $F$ and 3 objects $X$ in membrane of label " 3 " with negative charge. In the same step, by using $\mathrm{R}_{18}$, the membrane " 2 " is divided into two copies of neutral charge.

Now the charge of membranes with label " 3 " turn to neutral again, and according to the priority relationship $\mathrm{R}_{19}>\mathrm{R}_{21}>\mathrm{R}_{24}>\mathrm{R}_{28}, \mathrm{R}_{19}$ is the next rule to be used. $\mathrm{By}$ using $\mathrm{R}_{19}$, the number of the object $F$ in those two membrane of label " 3 " are decreased to $a^{2}(\bmod p)$ and $a^{3}(\bmod p)$ respectively. Then, $\mathrm{R}_{21}$ is used to transform $F G$ into $H$. If there is a membrane of label " 3 " which does not contain $F$ or $G$ after $\mathrm{R}_{21}$ has been applied, in other words, $a^{2} \equiv b(\bmod p)$ or $a^{3} \equiv b(\bmod p)$, this one will be dissolved by $\mathrm{R}_{25}$ and all the objects $X$ in it will be sent to the environment by $\mathrm{R}_{28}, \mathrm{R}_{29}$. Otherwise, the objects $F$ and $G$ would be changed back to $A$ by $\mathrm{R}_{26}$ and $\mathrm{R}_{27}$. Hence the rule $\mathrm{R}_{0}$ can be used again, and it is the beginning of the next loop.

In this way, in stage one of the $\mathrm{i}$-th $(1 \leq i \leq\lceil\log (p-1)\rceil)$ round, there would be $2^{\mathrm{i}}$ membranes of label " 3 " contained by a membrane of label " 2 ". And in each membrane " 2 " the objects $A$ of number $a^{j(\bmod p)(1<j<p-1)}$ would be sent into the membrane of label " 1 " and generate $\left.a^{2 j(} \bmod p\right)$ objects $\left.F\left(\text { note that }\left(a^{j(} \bmod p\right)\right)^{2(} \bmod p\right)$ $\left.\equiv a^{2 j}(\operatorname{modp})\right), 2 \mathrm{j}$ objects $X$ in membrane " 1 " of positive charge, $\left.a^{2 j+1(} \operatorname{modp}\right)$ objects $F, 2 \mathrm{j}+1$ objects $X$ in membrane " 1 " of negative charge. Then, through the operation in stage two and stage three, we have checked the value of the index from $2^{\mathrm{i}}$ to $2^{\mathrm{i}+1}-1$ in the $\mathrm{i}$-th round. Finally, we exhaustively search all the possible values of

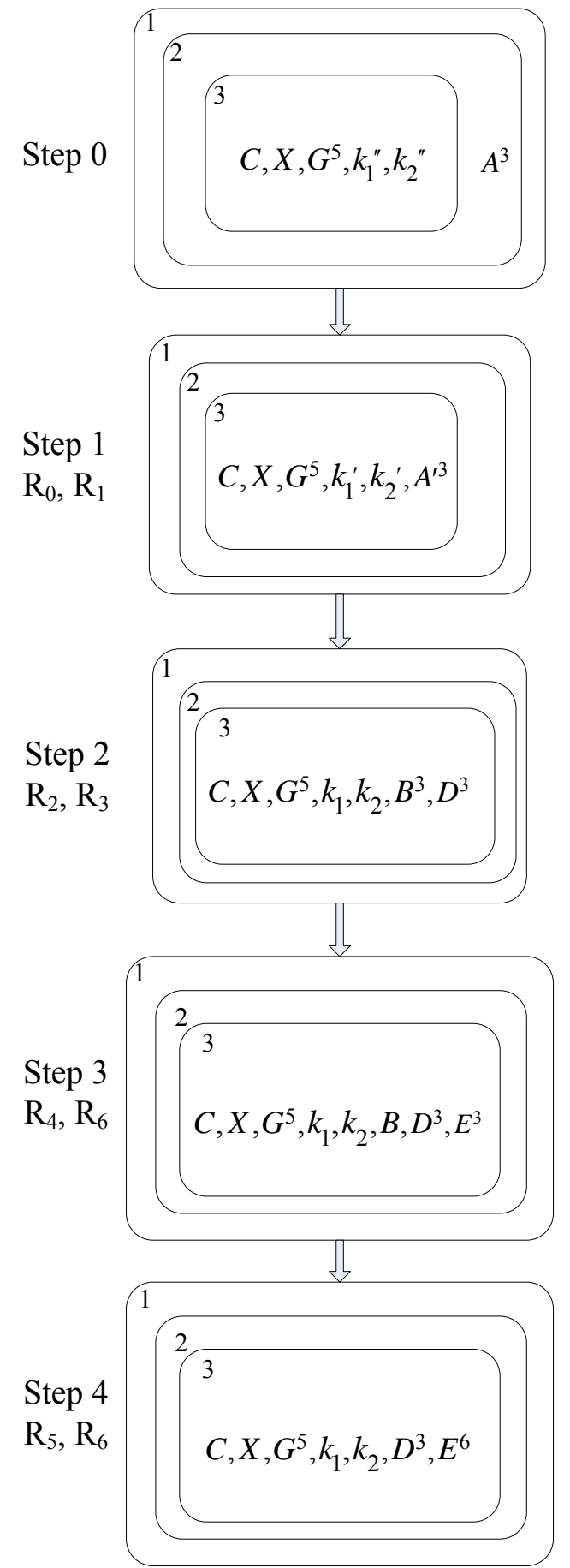

Figure 3. The evolution process of the P system solving the Discrete Logarithm Problem (step0 4)

$x$ and can get the correct index value for the DLP in less than $\lfloor\log (p-1)\rfloor$ loops.

To explain the evolution process more clearly but, more importantly, to prove this $\mathrm{P}$ system, we give an example, to calculate: $x=\log _{3} 5 \bmod 7 . a=3, b=5, p=7$.

We explain the evolution process as follows:

1. Step 0

In the initial configuration, there are five occurrences of object $G$ in the membrane of label 
"3" and three occurrences of object $A$ in the membrane of label " 2 ".

2. Step 1

According to the rules $\mathrm{R}_{0}$ and $\mathrm{R}_{1}$, all the three objects $A$ will go into the membrane " 3 " and evolve to the object $A^{\prime}$, and the objects $k_{1}^{\prime \prime}$ and $k_{2}^{\prime \prime}$ evolve to $k_{1}^{\prime}$ and $k_{2}^{\prime}$.

3. Step 2

Under $\mathrm{R}_{2}$ and $\mathrm{R}_{3}$ every object $A^{\prime}$ in the membrane " 1 " evolve to two objects $B$ and $D$, and the objects $k_{1}^{\prime}$ and $k_{2}^{\prime}$ evolve to $k_{1}$ and $k_{2}$.

4. Step 3

Two objects $B$ and one object $C$ evolve to one object $C$ under the evolution rule $\mathrm{R}_{4}$. The three occurrences of object $D$ produce three occurrences of object $E$ according to $\mathrm{R}_{6}$.

5. Step 4

According to the priority relationship $\mathrm{R}_{4,5}>\mathrm{R}_{7}$, the last object $B$ and the object $C$ evolve to one object $C$ under the evolution rule $R_{4}$. The three occurrences of object $D$ produce another three occurrences of object $E$ according to $\mathrm{R}_{6}$.

The "Step 0-4" of the evolution process solving this problem is presented in Fig.3. "Step 0" shows the initial configuration.

6. Step 5

There is no object $B$ left, so $\mathrm{R}_{7}$ can be used now. $\mathrm{R}_{7}$ divides the membrane " 3 " into two separate copies of opposite charge. At the same time, $\mathrm{R}_{6}$ produces another three occurrences of object $E$. Now there are nine occurrences of object $E$ in both of the membranes with label " 3 ".

7. Step 6

$\mathrm{R}_{8} \sim \mathrm{R}_{18}$ "absorb" the objects $D, t_{1}$, and $\mathrm{f}_{1}$, produce one object $Z^{\prime \prime}$ in both of the membranes with label " 3 ". What's more, the objects $X$ and $E$ evolve to two objects $X$ and nine objects $F$ in the membrane with positive charge, and three objects $X$ and 27 objects $F$ in the membrane with negative charge.

8. $\quad$ Step 7

$\mathrm{R}_{19}$ consumes the objects $F$, and the numbers of the objects $F$ left in the two membranes " 3 " are 2 and 6 , which are the remainder modulo $7 . \mathrm{R}_{20}$ transforms object $Z^{\prime \prime}$ to $Z^{\prime}$.

9. Step 8

$\mathrm{R}_{21}$ transforms the paired objects $G$ and $F$ into object $H . \mathrm{R}_{22}$ transforms object $Z^{\prime}$ to $Z$.

10. Step 9

Since there are object $G$ left in membrane "3" with positive charge and object $F$ left in membrane with negative charge, $R_{23}$ and $R_{24}$ delete the object $Z$ according to the priority relationship $R_{23,24}>R_{25}$. $R_{25}$ cannot be used to dissolve any membrane, and $\mathrm{R}_{26}$ transforms the object $H$ back to object $F$ and $G$, so the computation continues.

The "Step 5-9" of the evolution process solving this problem is presented in Fig.4.

11. Step 10
In this step, all the objects $F$ evolve to object $A$ and be sent out of each membrane " 3 " by $\mathrm{R}_{27}$. Thus, both membrane structures in membrane " 1 " are similar to the initial configuration, and the next loop begins.

12. Step $10 \sim \mathrm{n}$

These steps are similar to the step $0 \sim 7$. Now we get four membrane structures composed of membranes with label "2" and "3" $\left(\left[{ }_{2}[3]_{3}\right]_{2}\right)$. We focus on the second membrane structure in which the number of object $F$ is the same to the number of object $G$.

13. Step $\mathrm{n}+1$,

$\mathrm{R}_{21}$ transforms the paired objects $G$ and $F$ into object $H$. $\mathrm{R}_{22}$ transforms object $Z^{\prime}$ to $Z$.

14. Step $n+2$

There is no $F$ or $G$ left in the region " 3 ", so the rule $\mathrm{R}_{23}$ and $\mathrm{R}_{24}$ cannot be applied. According to the priority relationship $R_{25}>R_{26}, R_{25}$ is used in the second membrane structure. $\mathrm{R}_{25}$ dissolves the membrane, and release all the objects in region " 3 " to the region " 2 ".

15. Step $n+3$, Step $n+4$

$\mathrm{R}_{28}$ and $\mathrm{R}_{29}$ send $X$ to the environment. The number of the objects $X$ is the results: 5 .

The "Step 10-n+4" of the evolution process solving this problem is presented in Fig.5. Fig.6 shows the whole evolution process of membrane structure clearly.We can conclude that the Discrete Algorithm Problem can be solved in at most two loops for $p=7$. 


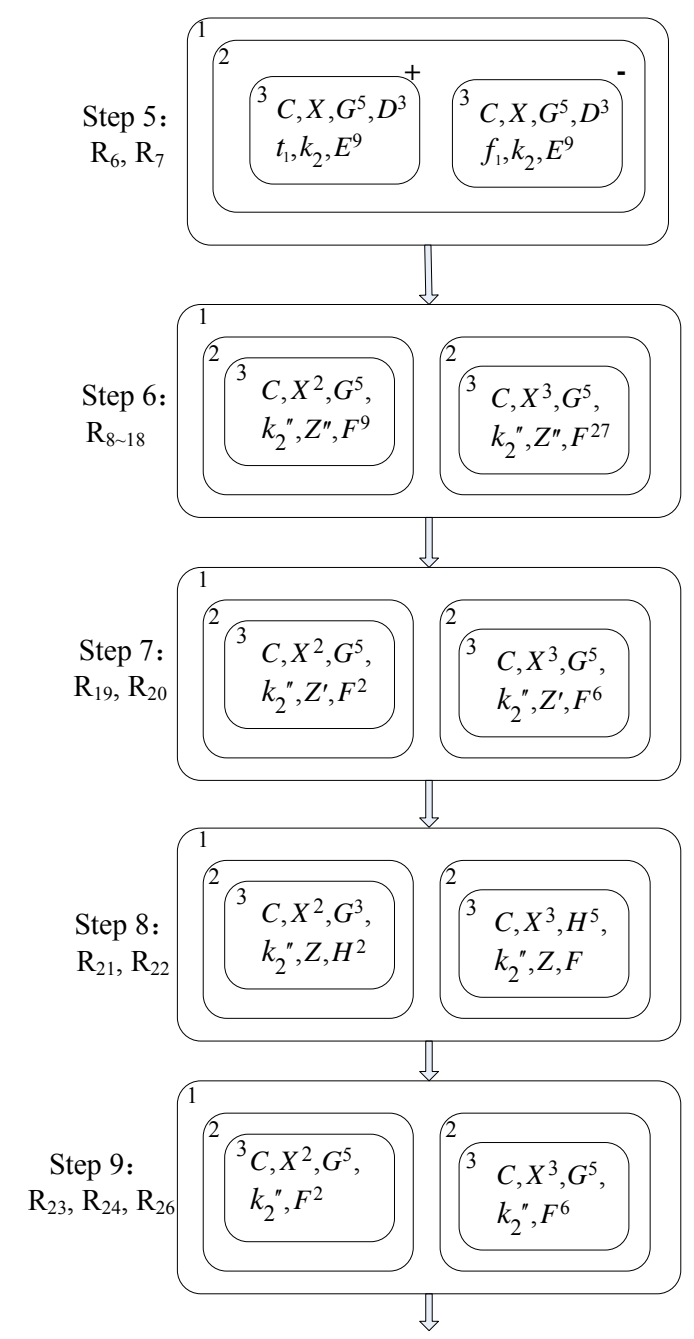

Figure 4. The evolution process of the P system solving the Discrete Logarithm Problem (step5 9)

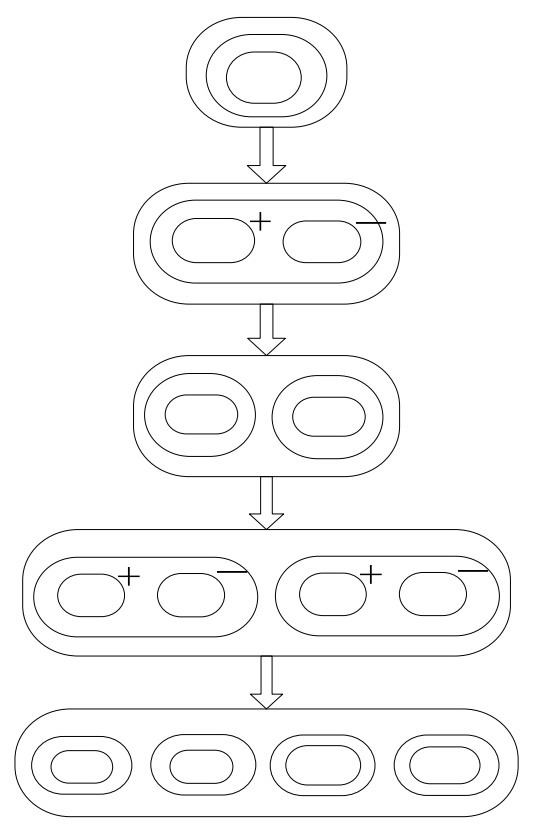

Figure 6. The evolution process of the P system solving the Discrete I nogarithm Prohlem

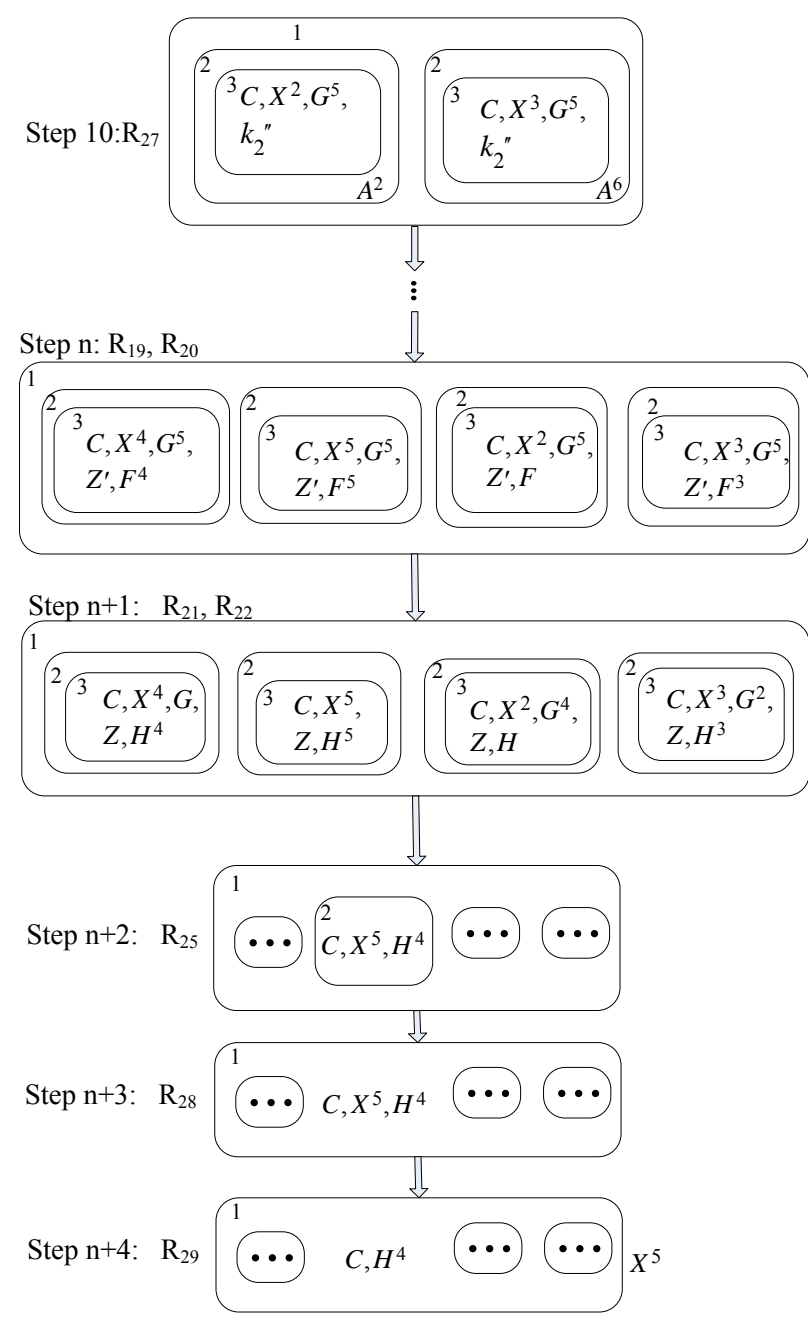

Figure 5. The evolution process of the P system solving the Discrete Logarithm Problem (step10 n+4)

\section{CONCLUSION}

We present a $\mathrm{P}$ system with active membranes and strong priority to solve the Discrete Logarithm Problem used in Diffie-Hellman key exchange protocol for the first time. In future work, we will further improve the performance of this system.

\section{ACKNOWLEDGMENT}

This work is supported by the National Natural Science Foundation of China under Grand No. 60573120, and by the National High Technology Research and Development Program of China(863 Program) under Grand No. 2007AA01Z420, and by the Key project sponsored by Natural Science Foundation of Hubei Province under Grand No. 2008CDA021.

\section{REFERENCES}

[1] G. Paun, "Computing with Membranes," Journal of Computer and System Sciences, vol. 61, 2000, pp. 108-143.

[2] "Home - The P Systems Webpage"; 
http://ppage.psystems.eu/.

[3] W. Diffie and M.E. Hellman, "Multiuser cryptographic techniques," IEEE Transactions on Information Theory, 1976.

[4] P.W. Shor, "Polynomial-Time Algorithms for Prime Factorization and Discrete Logarithms on a Quantum Computer" SIAM J.Sci.Statist.Comput. 26 (1997) 1484

[5] G. Ciobanu, G.P. aun, and M.J. Pérez-Jiménez, eds., Applications of Membrane Computing, Springer-Verlag, 2005.

[6] A. Obtulowicz, "On P systems with Active Membranes Solving Integer Factorizing Problem in a Polynomial Time," C. Calude et al., ed., Springer-Verlag, 2001, p. 267-286.

[7] G. Paun, "P systems with active membranes: attacking NPcomplete problems," Journal of Automata, Languages and Combinatorics, vol. 6, 2001, pp. 75-90.

[8] G. Paun, Membrane Computing: An Introduction, Springer, 2002.

[9] Whit Diffie and Martin Hellman. New Directions in Cryptography. IEEE Transactions on Information Theory, IT-22(6):644-654, November 1976.

[10] A. Evans, Jr., W. Kantrowitz, and E. Weiss, A improved algorithm for computing algorithm in $G F(p)$ and its cryptographic significance. IEEE Transactions on Information Theory, vol. 24, 2003, pp. 106-110.

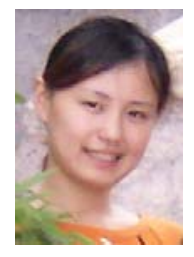

Xiaojing Ma was born in Jinzhai, China in 1983, and received her B.E. degree from Wuhan University of Science and Technology, Wuhan, China, 2003. She is currently a PhD student in the Department of Computer Science and Technology at Huazhong University of Science and Technology. Her research interests include: cryptography, network security and $\mathrm{P}$ systems.
She was a Software Engineer of 263 On-Line Communications Inc. She has published several papers in the areas of multicast key management and $\mathrm{P}$ systems.

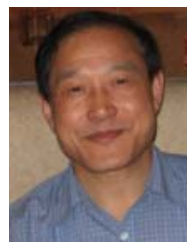

Zhitang Li was born in Jianli, China in 1951, and received his M.E. degree in Computer Architecture from Huazhong University of Science and Technology, Wuhan, China, 1987, and $\mathrm{PhD}$ degree in Computer Architecture from Huazhong University of Science and Technology, Wuhan, China, 1992. His research interests include computer architecture, network security and P2P networks.

He is currently the director of China Education and Research Network (CERNET) in Central China. He was a vice president of Department of Computer Science and Technology, Huazhong University of Science and Technology, China. He has published more than one hundred papers in the areas of computer architecture, network security and P2P networks.

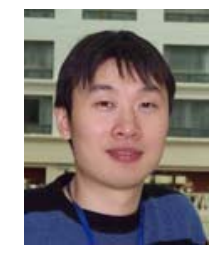

Hao Tu was born in Wuhan, China in 1977, and received his B.S. and $\mathrm{PhD}$ degrees in computer science and technology from Huazhong University of Science and Technology, Wuhan, China, in 1999 and 2008. His research interests include: computer and network security, network measurement.

He is currently a lecturer in the Network Center, Huazhong University of Science and Technology. 